\section{Stressing a role for Staufen 1 in SCA2 pathogenesis}

A new report in Nature Communications has identified a key role for the RNAbinding protein Staufen 1 (STAU1) in the pathogenesis of spinocerebellar ataxia type 2 (SCA2), a form of cerebellar ataxia caused by CAG repeat expansions in the ataxin 2 gene (ATXN2). STAU1 was found to contribute to dysregulation of RNA metabolism through an interaction with the ATXN2 protein.

"Since we identified the ATXN2 gene in 1996, we have worked to understand its function in neurodegeneration," explains study leader Stefan Pulst. "STAU1 was known to be involved in RNA transport and degradation, as well as in translational control, but a role in neurodegeneration had not been recognized."

Pathologically, SCA2 is characterized by accumulation of ATXN2 in stress granules, which are aggregates of RNA and protein that form under conditions of cellular stress and consist largely of stalled mRNA translation initiation complexes. Pulst and colleagues observed colocalization of STAU1 and ATXN2 in stress granules in fibroblasts from patients with SCA2 and in cerebellar Purkinje cells in an SCA2 mouse model.

The researchers also showed that STAU1 levels were elevated in cell lines from patients with SCA2 and in cerebellar extracts from SCA mice. This STAU1 overexpression was associated with aberrant processing of a variety of mRNAs, including PCP2, which is expressed in Purkinje cells and is depleted in SCA2. Reduction of STAU1 levels by RNA interference restored $P C P 2$ expression in cell lines. In addition, Stau 1 haploinsufficiency alleviated

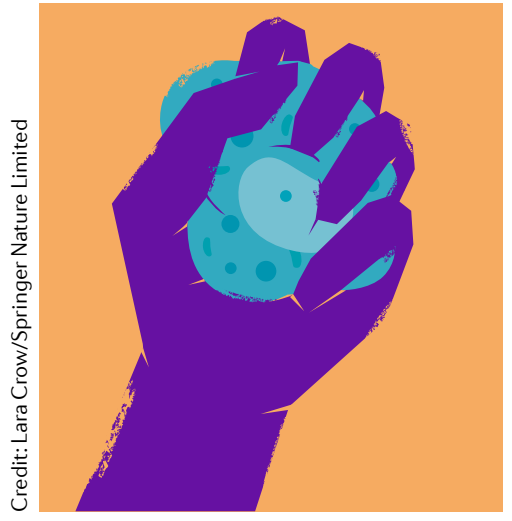

the pathological and behavioural phenotypes of SCA2 mice.

"We are working on understanding the precise mechanisms by which increased STAU1 abundance is detrimental to neurons," says Pulst. "We are also identifying antisense oligonucleotides to target STAU1 with the goal of testing these compounds in animal models of human neurodegenerative diseases, including amyotrophic lateral sclerosis."

Heather Wood

colocalization

of STAU 1 and

ATXN2 in stress granules

\title{
Do inflammatory profiles explain ALS-FTD spectrum?
}

Amyotrophic lateral sclerosis (ALS) and frontotemporal dementia (FTD) are thought to represent different manifestations along a single disease spectrum. A new study shows that neuroinflammation is associated with the symptomatic phase of ALS and FTD, and that different neuroinflammatory profiles could be one reason for the disparate presentations.

The study, led by Markus Otto, included a total of 151 participants, including people with genetic forms of ALS and FTD, people with sporadic ALS or FTD, nonsymptomatic carriers of mutations associated with ALS and FTD, and healthy controls. Cerebrospinal fluid (CSF) samples from all participants were analysed with enzyme-linked immunosorbent assays to measure the levels of the neuroinflammatory markers chitotriosidase 1 (CHIT1), chitinase-3-like protein 1 (CHI3L1, also known as YKL40) and glial fibrillary acidic protein (GFAP).

Levels of neuroinflammatory markers were unchanged in asymptomatic carriers of mutations associated with ALS and FTD, indicating that neuroinflammation is associated only with the symptomatic phases of these conditions.

Furthermore, estimates of changes in CSF marker levels over time indicated a sudden increase at the time of symptom onset.

"These findings suggest that neuroinflammation — as evaluated by this panel of established neuroinflammatory biomarkers - is either not an early event in ALS and FTD or [that] a different kind of neuroinflammation ... is taking place in the initial stages of these diseases," the authors write.

Neuroinflammatory markers were increased in patients with ALS and FTD, but the patterns differed. In ALS, levels of CHIT1 and YKL 40 were increased relative to those in controls, but levels of GFAP were unaffected. In FTD, levels of YKL40 and GFAP were increased, but levels of CHIT1 were barely affected. These patterns were consistent between the genetic and sporadic forms of the conditions.

The specific neuroinflammatory profiles indicate "more severe macrophage/microglia activation in ALS and astrocytosis in FTD," according to the authors. "GFAP determination in CSF might be an additional tool to improve identification of FTD in patients with ALS," they conclude. However, they also note that follow-up studies are needed to determine the diagnostic potential.

\section{lan Fyfe}

ORIGINAL ARTICLE Oeckl, P. et al. Different neuroinflammatory profile in amyotrophic lateral sclerosis and frontotemporal dementia is linked to the clinical phase. J. Neurol. Neurosurg. Psychiatry https://doi.org/10.1136/jnnp-2018-318868 (2018) 